\title{
Multi-ingredient pre-workout supplementation changes energy system contribution and improves performance during high-intensity intermittent exercise in physically active individuals: a double- blind and placebo controlled study
}

Caique Figueiredo ${ }^{1}$, Fábio Santos Lira ${ }^{1}$, Fabricio Eduardo Rossi ${ }^{2}$, François Billaut ${ }^{3}$, Rodrigo Loschi ${ }^{1,4}$ and

Camila S. Padilha ${ }^{1 *}$ (D)

\begin{abstract}
Background: Nutritional ergogenic aids are commonly used to boost physiological adaptations to exercise and promote greater fitness gains. However, there is a paucity of data about multi-ingredient pre-workout supplementation (MIPS). Therefore, the aim of the present study was to investigate the acute effects of MIPS on the oxidative, glycolytic and ATPCP energy systems contribution, time spent above $90 \% \dot{\mathrm{V}}_{2 \max }\left(T 90 \% \dot{\mathrm{V}} \mathrm{O}_{2 \text { max }}\right)$, excess post-exercise oxygen consumption (EPOC) magnitude, number of efforts and time to exhaustion during a high-intensity interval exercise (HIIE) session.

Methods: Twelve physically-active and healthy men completed the HIIE sessions that involved running bouts of $15 \mathrm{~s}$ on the treadmill at 120\% of the maximum aerobic speed (MAS), interspersed with $15 \mathrm{~s}$ of passive recovery. Blood lactate was collected at immediately post, 3, 5, and 7 min post exercise. The contribution of ATP-CP, glycolytic and oxidative systems was analyzed at rest, during the HIIE sessions and for 20 min post. Performance variables (time to exhaustion, number of efforts) and oxygen consumption were also analyzed.

Results: MIPS significantly increased the number of efforts performed (MIPS: $41 \pm 10$ vs Placebo: $36 \pm 12, p=0.0220$ ) and time to exhaustion (MIPS: $20.1 \pm 6$ min vs Placebo: $17 \pm 5 \mathrm{~min}, p=0.0226)$. There was no difference between supplements for both $\mathrm{T} 90 \% \dot{\mathrm{V}}_{2 \max }(p=0.9705)$ and EPOC $(p=0.4930)$. Consuming MIPS significantly increased the absolute oxidative energy system contribution by $23.8 \%(p=0.0163)$ and the absolute ATP-CP contribution by $28.4 \%(p=0.0055)$ compared to placebo. There was only a non-significant tendency for a higher glycolytic system contribution after MIPS ingestion $(p=0.0683)$.

(Continued on next page)
\end{abstract}

\footnotetext{
* Correspondence: camilapersonal@yahoo.com.br

${ }^{1}$ Exercise and Immunometabolism Research Group, Department of Physical Education, School of Technology and Sciences, Presidente Prudente, São Paulo, Brazil; Post Graduation Program in Physical Therapy, São Paulo State University (UNESP), São Paulo, Brazil

Full list of author information is available at the end of the article
}

(c) The Author(s). 2020 Open Access This article is licensed under a Creative Commons Attribution 4.0 International License, which permits use, sharing, adaptation, distribution and reproduction in any medium or format, as long as you give appropriate credit to the original author(s) and the source, provide a link to the Creative Commons licence, and indicate if changes were made. The images or other third party material in this article are included in the article's Creative Commons licence, unless indicated otherwise in a credit line to the material. If material is not included in the article's Creative Commons licence and your intended use is not permitted by statutory regulation or exceeds the permitted use, you will need to obtain permission directly from the copyright holder. To view a copy of this licence, visit http://creativecommons.org/licenses/by/4.0/. The Creative Commons Public Domain Dedication waiver (http://creativecommons.org/publicdomain/zero/1.0/) applies to the data made available in this article, unless otherwise stated in a credit line to the data. 
(Continued from previous page)

Conclusion: Acute MIPS ingestion appears effective at increasing both aerobic and anaerobic alactic energy contribution and time to exhaustion during a HIIE protocol.

Keywords: Pre-workout drink, Performance fitness, Energy expenditure, Ergogenic aid

\section{Background}

High-intensity intermittent exercise (HIIE) is an excellent strategy for increasing maximal oxygen uptake $\left(\dot{\mathrm{V}}_{2 \max }\right)$ and decreasing body fat $[1,2]$. HIIE protocols involving relatively short efforts $(<1 \mathrm{~min})$ performed at an intensity corresponding to or above $100 \%$ of maximal aerobic speed (MAS) have been frequently used to increase aerobic power [3, 4]. The effectiveness of HIIE is attributed to, at least in part, a higher energy expenditure and number of efforts near $\dot{\mathrm{VO}}_{2 \max }(90-100 \%)$. Indeed, these factors are important to increase cardiorespiratory fitness $[3,5]$. Higher cardiorespiratory fitness is related to higher physical performance and reduced risk of cardiovascular diseases $[6,7]$. Therefore, strategies that optimize energy expenditure during a HIIE session are extremely important for reducing excess body fat accumulation [8].

Nutritional ergogenic aids are commonly used to boost these physiological adaptations to exercise and to promote greater fitness gains. In this perspective, some exercise modalities have been combined with multi-ingredient preworkout supplementation (MIPS; typically containing creatine, arginine, leucine, valine, isoleucine, tyrosine and caffeine) to enhance sympathetic response, substrate availability, and muscle contraction [9-11]. Acute ingestion of MIPS has been demonstrated to optimize performance during continuous runs to exhaustion [11] and during tests of explosive power, anaerobic power and maximum strength [10]. Furthermore, resting energy expenditure, as well as perceptions of readiness to perform and cognitive function were also increased after MIPS [12]. In addition, the absolute energy expenditure may precede improvements in performance, since the higher ATP resynthesis rate is partly associated with increased enzyme activity, uptake of substrates/oxygen and/or removal of metabolites [13]. However, there is scarce evidence about the potential impact of MIPS on energy systems contribution and overall oxygen consumption during HIIE protocols, which are typically used by various populations to enhance fitness and body composition. Knowing which system is affected the most by MIPS ingestion could refine the prescription of this ergogenic aid to optimize specific training sessions and/or increase energy expenditure for particular populations. Therefore, there is a wide scope of research to be done in this area to promote both health and performance outcomes.

Our group recently investigated the physiological responses to HIIE ( $15 \mathrm{~s}$ of effort at the speed associated with $120 \% \dot{\mathrm{VO}}_{2 \max }$ interspersed with $15 \mathrm{~s}$ of passive recovery) after supplementation of a single ergogenic aid (capsaicin) that stimulates the sympathetic nervous system, lipid oxidation, and muscle contraction [14]. Results indicated no difference in the time spent above $90 \%$ of $\dot{\mathrm{V}}_{2 \max }\left(\mathrm{T} 90 \% \dot{\mathrm{V}}_{2 \max }\right)$ and energy systems contribution, although capsaicin significantly increased the number of efforts performed before volitional exhaustion of the participants. One may argue that using multiple ingredients could not only increase the number of efforts, and thereby the overall training load, but also raise energy expenditure and $\dot{\mathrm{VO}}_{2}$ during HIIE [14]. Therefore, the aim of the present study was to investigate the acute effects of MIPS on the oxidative, glycolytic and adenosine triphosphate - creatine phosphate (ATP-CP) energy systems contribution, $\mathrm{T} 90 \% \mathrm{~V}_{2 \max }$ and excess post-exercise oxygen consumption (EPOC) magnitude in a HIIE session. We hypothesized that the MIPS would increase $\mathrm{T} 90 \% \dot{\mathrm{VO}}_{2 \max }$ during HIIE and the EPOC after the session, conducive to an overall increase in energy expenditure.

\section{Methods \\ Study design}

This study used a randomized, double-blind, crossover design with experimental trials conducted in the morning (7 to $10 \mathrm{AM}$ ) and each visit separated by $72 \mathrm{~h}$. During the first visit, anthropometric measurements and the incremental running test was performed to determine $\dot{\mathrm{VO}}_{2 \max }$ and MAS. The following two visits, participants randomly consumed the MIPS or Placebo capsules one hour before performing a HIIE session. The $\dot{\mathrm{VO}}_{2}$ and blood lactate concentration were monitored during the HIIE sessions to determine metabolic effort.

\section{Subjects}

Twelve physically-active and healthy men were recruited for this study. The interview considered the following criteria of inclusion: being male, between 18 to 35 years age and practice aerobic exercise for at least 6 months. The participants were also excluded if they presented any medical contraindications that might interfere with their performance on the exercise protocol. All participants signed a consent form and were informed about the purpose of the study and the possible risks involved. This study was conducted after approval by the Ethics Research Group of the Federal University of Piauí, 
Teresina-PI, Brazil (Protocol number: 3.169.545) and according to the 2008 Revision of the Declaration of Helsinki [15]. The data collection process (CONSORT diagram) is shown in Fig. 1.

Dietary intake assessment, supplementation protocol and anthropometric measurement

Participants were instructed to consume the same breakfast and to record food and fluid intake $24 \mathrm{~h}$ before each HIIE session. Participants were also instructed to avoid coffee, tea, alcoholic beverages, or any other stimulants, supplement or ergogenic substance during the study period, and were instructed to maintain their regular food intake. The consumption of kilocalories and macronutrients was calculated based on the Brazilian food composition table (TACO) to ensure that intake was similar in both experimental trials.

The MIPS used in this study was a commercially available product supplied by the manufactuer (Supley Laboratório, Matão, São Paulo, Brazil). The ingredients are listed in Table 1. The Placebo mixture (maltodextrin + juice Clight $^{\circ}$ containing maltodextrin, dyes, acidulants and flavorings, energy value $=34 \mathrm{Kcal}$, carbohydrates $=8.5 \mathrm{~g}$ ) was diluted in $250 \mathrm{ml}$ of water. Both placebo and MIPS mixture were identical in color and flavor. The MIPS or Placebo were delivered to the volunteers by a member of the laboratory who did not belong to the investigating research team to ensure a double-blind design. Participants ingested the substance in the laboratory one hour before the start of the experimental HIIE session. Body weight was measured using an electronic scale (Filizola PL50,

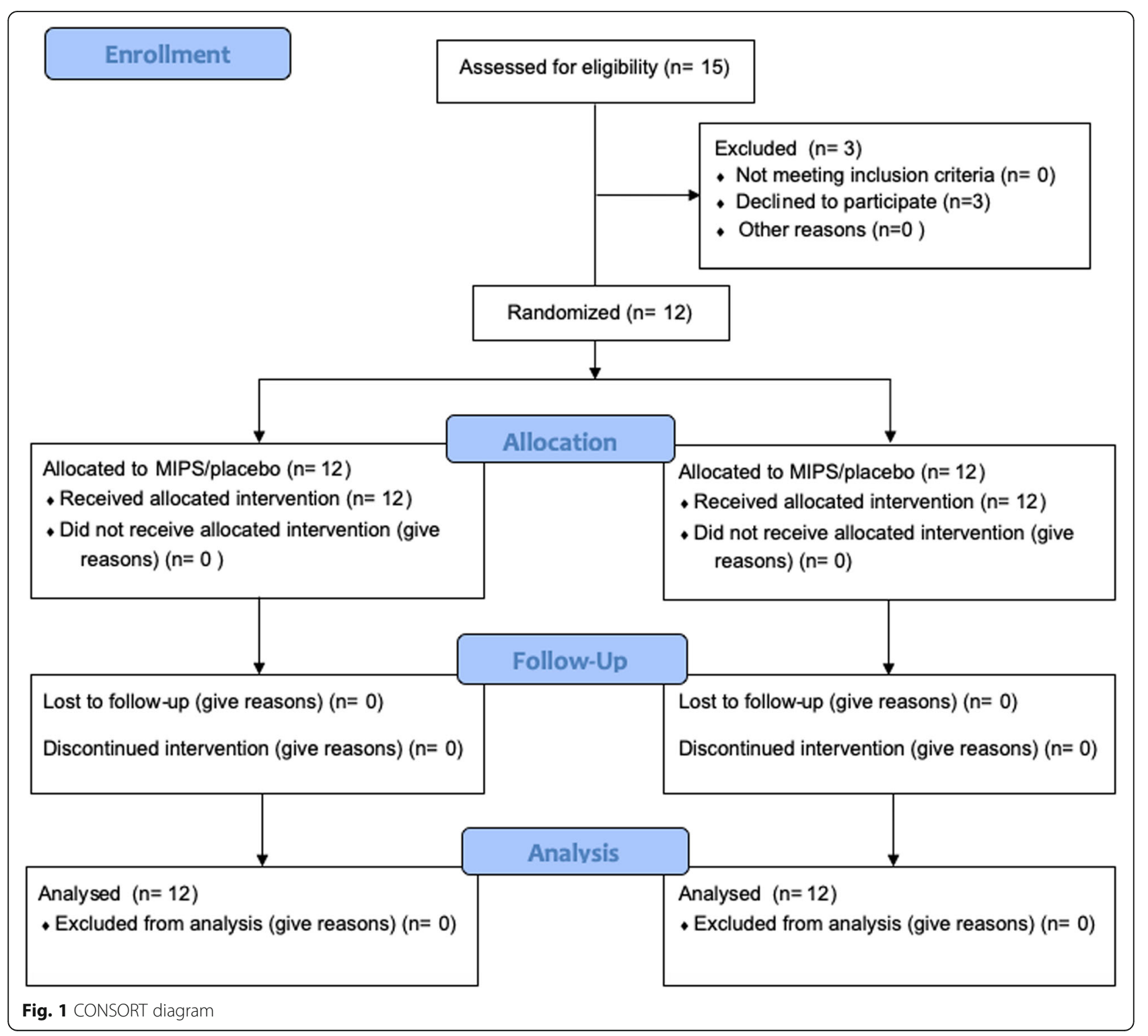


Table 1 Supplement ingredients

\begin{tabular}{|c|c|c|}
\hline \multicolumn{3}{|c|}{ Serving Size: 3 Scoops (20 g) } \\
\hline Amount Per Serving & Amount & \% Daily Value \\
\hline Calories & 71 & $4 \%$ \\
\hline Total Carbohydrates & $8 \mathrm{~g}$ & $3 \%$ \\
\hline Calcium & $180 \mathrm{mg}$ & $18 \%$ \\
\hline Folic acid & $500 \mathrm{mcg}$ & $208 \%$ \\
\hline Vitamin C & $250 \mathrm{mg}$ & $556 \%$ \\
\hline Niacin & $35 \mathrm{mg}$ & $219 \%$ \\
\hline Vitamin B1 & $2 \mathrm{mg}$ & $167 \%$ \\
\hline Biotin & 20 mcg & $67 \%$ \\
\hline Vitamin B6 & $50 \mathrm{mg}$ & $3.846 \%$ \\
\hline Vitamin B2 & $2 \mathrm{mg}$ & $154 \%$ \\
\hline Vitamin B12 & $9.94 \mathrm{mcg}$ & $414 \%$ \\
\hline Proprietary Blend ${ }^{a}$ & $10.200 \mathrm{mg}$ & \\
\hline Caffeine & $400 \mathrm{mg}$ & * \\
\hline Creatine monohydrate & $3.000 \mathrm{mg}$ & * \\
\hline L-Arginine & $2.000 \mathrm{mg}$ & * \\
\hline L-Leucine & $2.000 \mathrm{mg}$ & * \\
\hline L-Valine & $1.000 \mathrm{mg}$ & * \\
\hline L-Isoleucine & $1.000 \mathrm{mg}$ & * \\
\hline L-Tyrosine & $800 \mathrm{mg}$ & * \\
\hline
\end{tabular}

"Daily Value (DV) not established

Filizola Ltda., Brazil). A fixed stadiometer was used to measure height with an accuracy of $0.1 \mathrm{~cm}$.

\section{Incremental running test}

Participants performed a maximal incremental test on a treadmill (Inbramed MASTER CI, Inbrasport ${ }^{\circ}$, Porto Alegre, Brazil) in an environment with controlled temperature and humidity. Expired gases were collected breath-by-breath with a silicon mask connected to the gas analyzer (Quark PFT - Cosmed ${ }^{\circ}$, Rome, Italy). The maximal incremental test was used to determine MAS for the prescription of HIIE sessions and $\dot{\mathrm{VO}}_{2 \max }$ for the characterization of the sample. Participants performed a warm-up of $5 \mathrm{~min}$ walking at $5 \mathrm{~km} / \mathrm{h}$ before test. The initial test speed was set at $6 \mathrm{~km} / \mathrm{h}$ and increased by 1 $\mathrm{km} / \mathrm{h}$ every $2 \mathrm{~min}$. Treadmill inclination was maintained a $1 \%$ and the test was terminated when the participant reached voluntary exhaustion. Verbal encouragements were provided to ensure that every volunteer ran to exhaustion. The MAS was assumed as the final velocity of the test. When the participants failed to complete a stage, the speed was selected according to the following formula: $\mathrm{MAS}=$ complete final stage velocity $+[$ (time, in seconds, remaining in incomplete final stage / $120 \mathrm{~s}) * 1$ $\mathrm{km} . \mathrm{h}$ ] [16]. The rate of perceived exertion (RPE) and heart rate $(\mathrm{HR})$ were measured during the test using the
6-20 point Borg scale [17] and Polar S810i (Polar, Finland), respectively.

\section{High-intensity intermittent exercise (HIIE) protocol}

For each trial session, participants performed a warm-up at $50 \%$ of MAS for $5 \mathrm{~min}$. The HIIE involved running bouts of $15 \mathrm{~s}$ on the treadmill at $120 \%$ of the MAS, interspersed with $15 \mathrm{~s}$ of passive recovery. The inclination of the treadmill was maintained at $1 \%$ and the HIIE session was terminated when the volunteers achieved voluntary exhaustion. The total of sprint intervals performed and time to exhaustion under each condition were recorded.

\section{Blood lactate}

Twenty-five microliters of blood was collected from the volunteer's right ear lobe at rest, immediately post, and 3,5 and $7 \mathrm{~min}$ post-exercise. Lactate concentrations were obtained using the Yellow Spring 1.500 Sport lactate analyzer (Yellow Springs, USA). The delta lactate (highest value minus rest values - $\left[\Delta \mathrm{La}^{-}\right]$) was utilized to compare conditions.

\section{Oxygen uptake}

$\dot{\mathrm{V}} \mathrm{O}_{2}$ was measured at rest, during the HIIE sessions and for $20 \mathrm{~min}$ after the end of the sessions. For data treatment, the mean $\dot{\mathrm{VO}}_{2}$ during the HIIE session was analyzed for $15 \mathrm{~s}$ in every effort and every for pause. The T90\% $\dot{\mathrm{V}} \mathrm{O}_{2 \max }$ was determined from the average obtained in the 5-s periods due to the rapid changes in $\mathrm{VO}_{2}$ over time between sprint and pause periods in order to obtain greater sensitivity, and compared with the relative $\dot{\mathrm{V}}_{2 \max }$ values. EPOC was obtained by subtraction of $\mathrm{VO}_{2}$ from rest of the mean $\dot{\mathrm{VO}}_{2}$ of recovery [14].

\section{Energy expenditure}

The energy expenditure during the MIPS and Placebo trials was estimated from the contribution of the oxidative, glycolytic, and ATP-PC energy systems $[18,19]$. The contribution of the oxidative energy system was estimated by subtraction of the resting $\dot{\mathrm{VO}}_{2}$ from the mean $\dot{\mathrm{VO}}_{2}$ calculated over the complete HIIE. For glycolytic energy system contribution, the $\Delta\left[\mathrm{La}^{-}\right]$was converted into oxygen equivalents assuming that the accumulation of $1 \mathrm{mmol} / \mathrm{L}$ of lactate is equivalent to $3 \mathrm{mLO}_{2} / \mathrm{kg}$ body weight [19]. Finally, the sum of $\dot{\mathrm{VO}}_{2}$-time average during the HIIE recovery periods ( $\Sigma$ EPOC) subtracted by resting $\dot{\mathrm{VO}}_{2}$ was assumed for the contribution of ATP-CP [20-22]. In addition, the fast component of EPOC (i.e., estimated using $\dot{\mathrm{V}} \mathrm{O}_{2}$ kinetics as the product of $\dot{\mathrm{VO}}_{2}$ amplitude and time constant using a bi-exponential fit) was calculated for the last effort utilizing the software Origin version 2019 (OriginLab Corporation, Microcal, Massachusetts, USA) and added to $\Sigma E P O C$. The oxygen equivalents were 
converted into energy equivalents considering $20.92 \mathrm{~kJ}$ for each $1 \mathrm{~L}$ of $\mathrm{O}_{2}$ used [23].

\section{Statistical analysis}

A power analysis (Power $1-\beta=0.87$ ) was performed a priori in $G^{*}$ Power software and indicated that effects could be detected with twelve participants. Data were reported as mean and standard deviation (SD). Data normality was verified using the Shapiro-Wilk test. The number of efforts performed, time to exhaustion, time above $90 \% \quad \dot{\mathrm{V}} \mathrm{O}_{2 \max }$, EPOC, dietary intake and oxidative, glycolytic and ATP-CP system contributions during MIPS and Placebo were analyzed by a paired $\mathrm{t}$-test. For the lactate, a two way analysis of variance [group (MIPS versus Placebo) $\times$ time point (pre versus post)] was conducted followed by the Tukey's post hoc test. Statistical significance was set at $p<0.05$. The data were analyzed using SPSS version 22.0 (SPSS Inc., Chicago, IL).

\section{Results}

Figure 2 shows the number of efforts performed, time to exhaustion, $\mathrm{T} 90 \% \dot{\mathrm{VO}}_{2 \max }$ (seconds), EPOC $20 \mathrm{~min}$ and lactate concentrations of each experimental trial. Consuming MIPS significantly increased the number of efforts performed (MIPS: $41 \pm 10$ vs Placebo: $36 \pm 12$, $p=0.0220$ ) and time to exhaustion (MIPS: $20.1 \pm 6 \mathrm{~min}$ vs Placebo: $17 \pm 5 \mathrm{~min}, p=0.0226$ ). There was no difference between MIPS and Placebo for both the T90\% $\dot{\mathrm{V}} \mathrm{O}_{2 \max }(p=0.9705)$ and EPOC $20 \mathrm{~min}(p=0.4930)$. On the other hand, the $\dot{\mathrm{V}}_{2 \max }$ was lower in the MIPS condition when the number of efforts were equal between MIPS and placebo conditions. Although $\left[\mathrm{La}^{-}\right]$increased at all moments post-exercise compared with pre-exercise, there was no significant difference between supplements $(p>$ 0.05).

Table 2 displays the dietary intake and macronutrient distribution $24 \mathrm{~h}$ before each trial. There was no difference in the dietary intake and macronutrient distribution of carbohydrate, protein and lipid $(p>0.05)$ of each experimental trial.

Table 3 displays the relative and absolute energy system contribution for each condition. Consuming MIPS significantly increased the absolute oxidative energy system contribution by $23.8 \%(p=0.0163)$ and the absolute ATP-CP contribution by $28.4 \%$ ( $p=$ $0.0055)$ related to placebo condition. However, we only noted a tendency for a greater glycolytic system contribution after MIPS ingestion during the HIIE session $(p=0.0683)$.

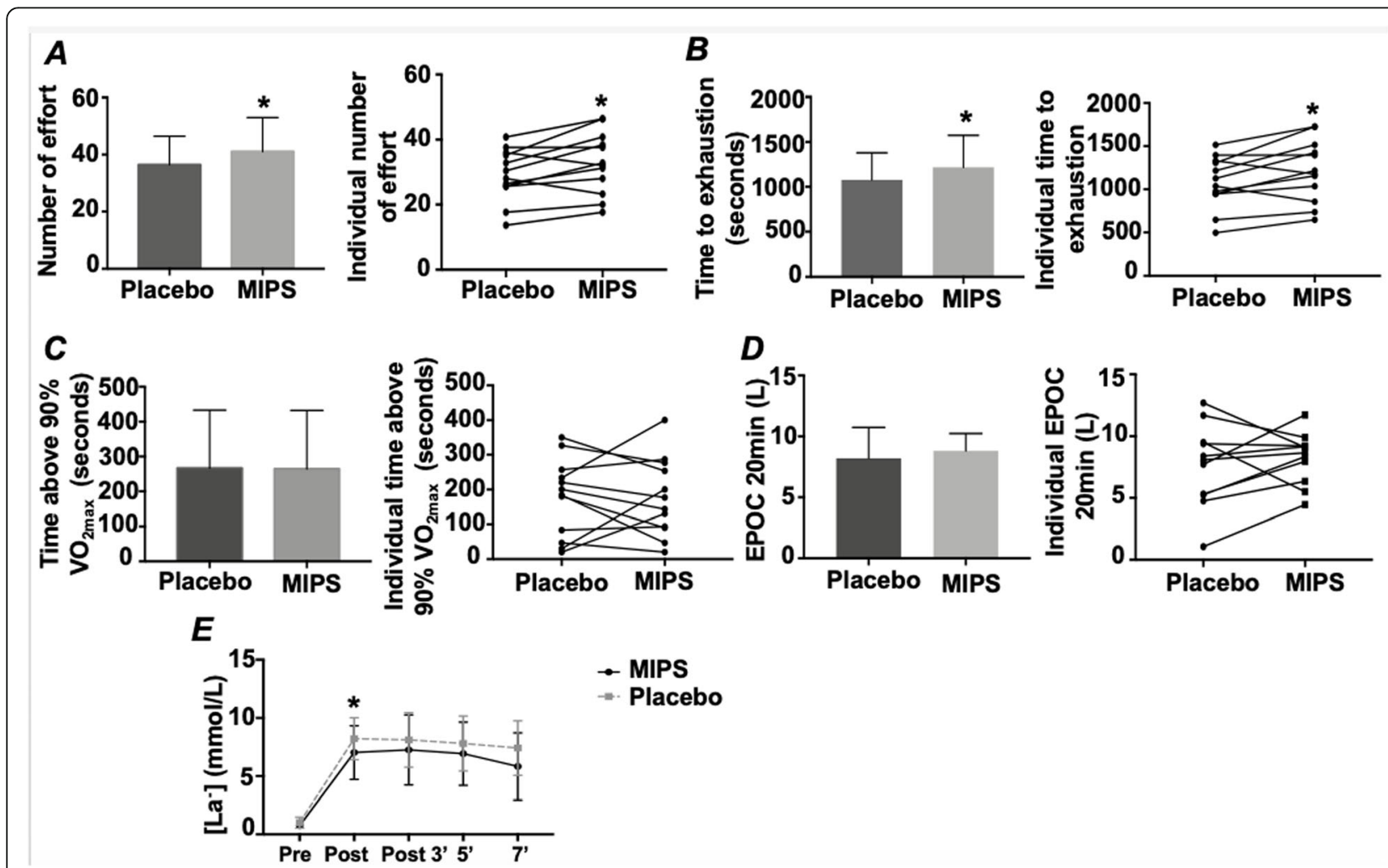

Fig. 2 Performance responses in MIPS and Placebo condition. Number of efforts performed (a), time to exhaustion (seconds) (b), time spent above $90 \%$ of the $\dot{V} \mathrm{O}_{2 \max }$ (seconds) (c), EPOC $20 \mathrm{~min}(\mathrm{~L})$ (d) and lactate concentration (mmol/L) at pre, post, post 3, 5 and $7 \mathrm{~min}(\mathbf{e})$ in MIPS and Placebo condition. Data are presented as mean \pm SD and individual response 
Table 2 Dietary intake and macronutrient distribution $24 \mathrm{~h}$ before each trial

\begin{tabular}{llll}
\hline Dietary intake & Placebo & MIPS & $p$ \\
\hline Carbohydrate $(\mathrm{g})$ & $281.7 \pm 133.7$ & $250.6 \pm 139.9$ & 0.148 \\
Carbohydrate $(\mathrm{g} / \mathrm{kg})$ & $3.7 \pm 1.8$ & $3.7 \pm 1.9$ & 0.151 \\
Protein $(\mathrm{g})$ & $122.6 \pm 36.6$ & $115.3 \pm 25.1$ & 0.701 \\
Protein $(\mathrm{g} / \mathrm{kg})$ & $1.6 \pm 0.4$ & $1.5 \pm 0.3$ & 0.769 \\
Lipid $(\mathrm{g})$ & $64.6 \pm 30.4$ & $66.4 \pm 25.4$ & 0.370 \\
Lipid $(\mathrm{g} / \mathrm{kg})$ & $0.8 \pm 0.4$ & $0.9 \pm 0.4$ & 0.455 \\
Total intake $(\mathrm{Kcal})$ & $2.361 \pm 895$ & $2.127 \pm 798$ & 0.394 \\
Total intake $(\mathrm{Kcal} / \mathrm{kg})$ & $31 \pm 12$ & $31 \pm 11$ & 0.459 \\
\hline
\end{tabular}

Data are presented as mean \pm SD. ${ }^{*} p=<0.05$ vs placebo condition

\section{Discussion}

To our best knowledge, this is the first study to investigate the acute effects of MIPS consumption on key physiological responses and performance during HIIE. The main findings of this study were that consuming MIPS one hour before exercise significantly increased time to exhaustion during 15-s running bouts at $120 \%$ MAS, and upregulated energy expenditure. However, our initial hypothesis that $\mathrm{T} 90 \% \dot{\mathrm{VO}}_{2 \max }$, EPOC, and glycolytic contribution would increase during HIIE after MIPS consumption was refuted.

Minimal research exists on the effects of MIPS on time to exhaustion [9]. In the present study, healthy male participants were able to perform 5 more efforts after ingesting $250 \mathrm{~mL}$ of MIPS compared to an isocaloric placebo. Our results are in accordance with a previous study showing that MIPS (Carnosyn ${ }^{\bullet}$ Patented Beta Alanine, Choline Bitartrate, L-Tyrosine, L-Glycine, Taurine, L-Carnitine Base, Beet Root Extract (Beta vulgaris) (High in Nitrates), Hawthorn Berry Powder (Crataegus pinnatifida) (Fruit), Agmatine Sulfate, Caffeine Anhydrous, Huperzine A 1\% (Huperzia serrata) significantly increased the total distance covered during a 25-s maximal running test on a treadmill (MIPS: $94.0 \pm 6.7$ vs placebo: $93.01 \pm 7.5 \mathrm{~m}, p=0.039)$ [24]. In addition, acute ingestion of MIPS ( $26 \mathrm{~g}$ of a powder containing an energy matrix (2.05 g of caffeine, taurine, glucuronolactone), a proprietary amino acid matrix $(7.9 \mathrm{~g}$ of L-leucine, Lisoleucine, L-valine, L-arginine and L-glutamine), $5 \mathrm{~g}$ of di-creatine citrate, and $2.5 \mathrm{~g}$ of $\beta$-alanine) mixed with 500 $\mathrm{mL}$ of water during continuous $3 \mathrm{~h}$ post-absorptive state, also increased exhaustion time by $12.5 \%$ compared to a placebo during an continuous exercise at $70 \%$ of $\dot{\mathrm{VO}}_{2 \max }$ on a treadmill [25]. Interestingly, the MIPS of all the studies presented above use caffeine as their "flagship" ingredient likely due to its potent and well-known acute ergogenic effects [26]. It has been robustly demonstrated that caffeine positively influences in central fatigue and muscular endurance by its direct effect on muscle anaerobic energy provision and its ability to increase muscle contractility [27, 28]. Caffeine acts as an adenosine receptor antagonist and has been shown to acutely improve cognition as well as performance during endurance, power, and resistance exercise when consumed in dosages between 3 and $6 \mathrm{mg} / \mathrm{kg}$ bodyweight $[9,29]$. The caffeine content of this MIPS (400 mg) is in that acceptable range for most individuals. A recent systematic review and meta-analysis of forty-six studies about caffeine reported a small but clear effect on endurance performance when taken in moderate doses $(3-6 \mathrm{mg} / \mathrm{kg})$ as well as an overall improvement in mean power output $(3.03 \pm 3.07 \%$; effect size $=0.23 \pm 0.15)$ and time-trial completion time $(2.22 \pm$ $2.59 \%$; effect size $=0.41 \pm 0.2$ ) [28]. Therefore, the caffeine contained in the MIPS tested in the present study may likely have contributed to the enhancement in performance at high intensity.

Tyrosine is a nonessential amino acid which plays an essential role in production of catecholamine neurotransmitters, including epinephrine, dopamine and norepinephrine. Coull et al., (2015) reported that tyrosine supplementation improves cognitive function during a simulated intermittent soccer performance test (iSPT),

Table 3 Energy system contribution for each condition

\begin{tabular}{|c|c|c|c|c|}
\hline & \multicolumn{2}{|l|}{ Placebo } & \multicolumn{2}{|l|}{ MIPS } \\
\hline & Mean \pm SD & $\begin{array}{l}\text { Lower - Upper } \\
95 \% \mathrm{Cl}\end{array}$ & Mean \pm SD & $\begin{array}{l}\text { Lower - Upper } \\
95 \% \mathrm{Cl}\end{array}$ \\
\hline \multicolumn{5}{|l|}{ Relative } \\
\hline Oxidative (\%) & $67.4 \pm 2.4$ & $65.0-73.7$ & $67.2 \pm 1.2$ & $64.5-69.1$ \\
\hline Glycolytic (\%) & $1.1 \pm 0.8$ & $0.4-2.8$ & $0.8 \pm 0.7$ & $0.5-2.6$ \\
\hline Phosphagen (\%) & $31.7 \pm 2.1$ & $25.9-33.8$ & $31.9 \pm 1.0$ & $30.1-33.6$ \\
\hline \multicolumn{5}{|l|}{ Absolute } \\
\hline Oxidative (Kcal) & $457.4 \pm 156.2$ & $250.2-687.0$ & $565.9 \pm 168.7^{*}$ & $296.8-810.1$ \\
\hline Glycolytic (Kcal) & $7.4 \pm 3.0$ & $3.9-12.2$ & $8.2 \pm 2.5$ & $5.2-11.9$ \\
\hline Phosphagen (kcal) & $210.7 \pm 64.0$ & $117.1-307.6$ & $271.0 \pm 80.1^{*}$ & $145.6-380.0$ \\
\hline
\end{tabular}

Data are presented as mean \pm SD and lower-upper $95 \% \mathrm{Cl}$ in placebo and MIPS conditions. ${ }^{*} p=<0.05$ vs placebo 
with no change in performance response [30]. Tyrosine supplementation alone seems to have little influence on performance, however, its cognitive action associated with other ergogenic resources deserves attention. The B-vitamins (thiamin, riboflavin, niacin, vitamin B6 and vitamin B12) are necessary in the energy-producing pathways of the body, while folate and vitamin B12 are required for the synthesis of new cells, such as the red blood cells, and for the repair of damaged cells [31]. Therefore, individuals with a vitamin deficit in the diet may be favored with consumption of MIPS, even though this is not the main objective of supplementation. On the other hand, athletes who have poor diets, those restricting energy intakes or eliminating food groups from the diet, should consider L-arginine supplementing. $\mathrm{L}$-arginine is an amino acid that is a precursor required for the synthesis of nitric oxide [32]. L-arginine would therefore favor muscle blood perfusion and increase of nutrient availability to muscle, and favor greater release of metabolites, such as lactate and ammnonia, which are related to the muscle fatigue [33]. However, almost all studies in trained athletes reported limited efficacy in improving blood flow or exercise performance with acute and chronic arginine oral supplementation [33]. Another important compound associated to performance is the creatine monohydrate. Although the acute effect of creatine supplementation be unknown, the supplementation of creatine monohydrate over time is able to increase performance in high intensity exercises, prevent injuries and help in the recovery process [34]. In addition, the effects of creatine monohydrate have been studied as a possible therapeutic strategy in neurodegenerative, metabolic and behavioral diseases [34]. Thus, although MIPS has these ingredients, they probably played a marginal role in the results found due to acute supplementation and caffeine is probably the only ingredient that may have contributed.

The energy contribution of HIIE protocols varies according to ATP demand and oxygen availability. The contribution of the aerobic system in the present study was greater than that of the anaerobic system $(67 \%$ vs $33 \%$ for MIPS condition). These relative results are in agreement with studies using the same [14] and a similar [22] protocol. More importantly, the MIPS in the present study was effective at increasing the oxidative and ATP-CP contribution, and there was also a tendency for an increased glycolytic contribution $(p=0.068)$. The study conducted by de Freitas et al. (2019) also used a protocol involving 15-s repetitions at 120\% MAS, with capsaicin supplementation, a natural bioactive substance that stimulates the sympathetic nervous system, lipid oxidation, and muscle contraction. This study did not observe any change in energy system expenditure, EPOC and $\mathrm{T} 90 \% \dot{\mathrm{V}}_{2 \max }$, despite a longer time of exhaustion with capsaicin $(+14.6 \%$ efforts). It is interesting to note that, although using different participants with probably different fitness levels, the number of efforts performed after ingesting a single dose of capsaicin was higher than after consuming MIPS (+13.8\% efforts) as done in the present study. However, the higher performance with capsaicin did not reflect a higher metabolic demand [14]. Thus, although the present study did not show significant changes in EPOC and T90\% MAS after MIPS ingestion, our initial hypothesis that MIPS could be a relevant strategy to increase energy expenditure is confirmed based on the findings of greater contribution of the oxidative system. These results are therefore of interest to people who wish to increase energy expenditure in HIIE sessions. The chronic effects of MIPS ingestion on body fat and cardiorespiratory fitness will have to be ascertained, especially in overweight populations. Furthermore, applied sport science studies will be required to explore the impact of MIPS in different sports and activity modalities.

The main limitation of the study was the absence of comparison between the MIPS and caffeine alone. In fact, although MIPS appears ergogenic compared to a placebo, a caffeine-equivalent control group would have ruled-out the impact of this substance alone vs. others and strengthened our understanding of the efficacy of MIPS. Nonetheless, based on these data, there is a need to explore the potency of combining MIPS with highintensity interval training to assess the chronic effect of this type of supplementation.

\section{Conclusion}

Acute ingestion of a multi-ingredient supplement containing vitamins, amino acids and caffeine is a relevant strategy to increase energy expenditure and time to exhaustion during HIIE.

\section{Abbreviations \\ ATP: Adenosine triphosphate; ATP-CP: Adenosine triphosphate - creatine phosphate; CAPES: Coordenação de Aperfeiçoamento de Pessoal de Nível Superior; EPOC: Excess post-exercise oxygen consumption; HIIE: High- intensity intermittent exercise; HR: Heart rate; La ${ }^{-}$: Lactate; MAS: Maximal aerobic speed; MIPS: Multi-ingredient pre-workout supplementation; RPE: Rate of perceived exertion; SD: Standard deviation; TACO: Brazilian food composition table; $\dot{V}_{2 \text { max }}$ : Maximal oxygen uptake}

\section{Acknowledgments}

We would like to thank all of subjects who participated in this study as well as the dietician supervision from Rodolfo Zanin.

\section{Authors' contributions}

CF, FSL and CSP designed the study. CF, FSL and CSP participated in data collection, contributed to analysis and interpretation of data. CF, FSL, FER, FB, $\mathrm{RL}$ and CSP assisted in the preparation of the manuscript. CF, FSL, FER, FB, RL and CSP wrote the initial draft of the manuscript. CF, FSL, FER, FB, RL and CSP critically reviewed the manuscript. All authors approved the final version of the manuscript and agree to be accountable for all aspects of the work in ensuring that questions related to the accuracy or integrity of any part of the work are appropriately investigated and resolved. 


\section{Funding}

This study was financed in part by the Coordenação de Aperfeiçoamento de Pessoal de Nível Superior - Brasil (CAPES) - Finance Code 001. We would like to thank Supley Laboratory for providing the supplement and placebo products that allowed us to conduct the study. Supley Laboratory is the manufacture of Proprietary Blend*, Matão, São Paulo, Brazil. The funding agencies CAPES and Supley Laboratory had no role in the experimental design used, data collection, and interpretation of results or concluding statements.

\section{Availability of data and materials}

Data and publication materials can be provided upon request. Please contact corresponding author for this information.

\section{Ethics approval and consent to participate}

Ethics approval for this study was approved by Ethics Research Group of the Federal University of Piauí, Teresina-PI, Brazil (Protocol number: 3.169.545). All participants provided written consent before participating in the study.

\section{Consent for publication}

Not applicable.

\section{Competing interests}

CF, FSL, FER, FB, and CSP declare no competing interest and Rodrigo Losh serves as scientific support of Supley laboratory.

\section{Author details}

${ }^{1}$ Exercise and Immunometabolism Research Group, Department of Physical Education, School of Technology and Sciences, Presidente Prudente, São Paulo, Brazil; Post Graduation Program in Physical Therapy, São Paulo State University (UNESP), São Paulo, Brazil. ${ }^{2}$ Immunometabolism of Skeletal Muscle and Exercise Research Group, Department of Physical Education and Associate Graduate Program in Health Science, Federal University of Piauí (UFPI), Teresina, PI, Brazil. Department of Kinesiology, Laval University, Laval, Quebec, QC, Canada. ${ }^{4}$ VP Centro de Nutrição funcional, São Paulo, Brazil.

Received: 21 January 2020 Accepted: 19 May 2020

Published online: 03 June 2020

\section{References}

1. Sultana RN, Sabag A, Keating SE, Johnson NA. The effect of low-volume highintensity interval training on body composition and cardiorespiratory fitness: a systematic review and meta-analysis. Sports Med. 2019;49(11):1687-721.

2. Keating SE, Johnson NA, Mielke Gl, Coombes JS. A systematic review and meta-analysis of interval training versus moderate-intensity continuous training on body adiposity. Obes Rev. 2017;18(8):943-64.

3. Buchheit M, Laursen PB. High-intensity interval training, solutions to the programming puzzle: part I: cardiopulmonary emphasis. Sports Med. 2013; 43(5):313-38.

4. Billat VL, Slawinksi J, Bocquet V, Chassaing P, Demarle A, Koralsztein JP. Very short (15s-15s) interval-training around the critical velocity allows middle-aged runners to maintain VO2 max for 14 minutes. Int J Sports Med. 2001;22(3):201-8.

5. Buchheit M, Laursen PB. High-intensity interval training, solutions to the programming puzzle. Part II: anaerobic energy, neuromuscular load and practical applications. Sports Med. 2013:43(10):927-54

6. Myers J, McAuley P, Lavie CJ, Despres JP, Arena R, Kokkinos P. Physical activity and cardiorespiratory fitness as major markers of cardiovascular risk: their independent and interwoven importance to health status. Prog Cardiovasc Dis. 2015:57(4):306-14

7. Bassett DR, Howley ET. Limiting factors for maximum oxygen uptake and determinants of endurance performance. Med Sci Sports Exerc. 2000;32(1):70-84.

8. Ng M, Fleming T, Robinson M, Thomson B, Graetz N, Margono C, et al. Global, regional, and national prevalence of overweight and obesity in children and adults during 1980-2013: a systematic analysis for the global burden of disease study 2013. Lancet. 2014;384(9945):766-81.

9. Harty PS, Zabriskie HA, Erickson JL, Molling PE, Kerksick CM, Jagim AR. Multiingredient pre-workout supplements, safety implications, and performance outcomes: a brief review. J Int Soc Sports Nutr. 2018;15(1):41.

10. Martinez N, Campbell B, Franek M, Buchanan L, Colquhoun R. The effect of acute pre-workout supplementation on power and strength performance. J Int Soc Sports Nutr. 2016;13:29.
11. Fukuda DH, Smith AE, Kendall KL, Stout JR. The possible combinatory effects of acute consumption of caffeine, creatine, and amino acids on the improvement of anaerobic running performance in humans. Nutr Res. 2010;30(9):607-14.

12. Jung YP, Earnest CP, Koozehchian M, Galvan E, Dalton R, Walker D, et al. Effects of acute ingestion of a pre-workout dietary supplement with and without. J Int Soc Sports Nutr. 2017;14:3.

13. Hara KY, Kondo A. ATP regulation in bioproduction. Microb Cell Factories. 2015;14:198.

14. de Freitas MC, Billaut F, VLG P, Rossi FE, Fiqueiredo C, Caperuto EC, et al. Capsaicin supplementation increases time to exhaustion in high-intensity intermittent exercise without modifying metabolic responses in physically active men. Eur J Appl Physiol. 2019;119:971-79.

15. Association WM. World medical association declaration of Helsinki: ethical principles for medical research involving human subjects. JAMA. 2013;310(20):2191-4.

16. Kuipers H, Verstappen FT, Keizer HA, Geurten P, van Kranenburg G. Variability of aerobic performance in the laboratory and its physiologic correlates. Int J Sports Med. 1985;6(4):197-201.

17. Borg G, Hassmen $P$, Lagerstrom M. Perceived exertion related to heart rate and blood lactate during arm and leg exercise. Eur J Appl Physiol Occup Physiol. 1987;56(6):679-85.

18. Margaria R, Edwards HT, Dill DB. The possible mechanisms of contracting and paying the oxygen debt and the rôle of lactic acid in muscular contraction. Am J Physiol Legacy Content. 1933;106(3):26.

19. di Prampero PE, Ferretti G. The energetics of anaerobic muscle metabolism: a reappraisal of older and recent concepts. Respir Physiol. 1999;118(2-3):103-15.

20. Zagatto A, Redkva P, Loures J, Kalva Filho C, Franco V, Kaminagakura E, et al. Anaerobic contribution during maximal anaerobic running test: correlation with maximal accumulated oxygen deficit. Scand J Med Sci Sports. 2011;21(6):e222-30.

21. Milioni F, Zagatto AM, Barbieri RA, Andrade VL, Dos Santos JW, Gobatto CA, et al. Energy systems contribution in the running-based anaerobic Sprint test. Int J Sports Med. 2017;38(3):226-32.

22. Panissa VLG, Fukuda DH, Caldeira RS, Gerosa-Neto J, Lira FS, Zagatto AM, et al. Is oxygen uptake measurement enough to estimate energy expenditure during high-intensity intermittent exercise? Quantification of Anaerobic Contribution by Different Methods. Front Physiol. 2018:9:868.

23. Gastin PB. Energy system interaction and relative contribution during maximal exercise. Sports Med. 2001:31(10):725-41.

24. Cameron M, Camic CL, Doberstein S, Erickson JL, Jagim AR. The acute effects of a multi-ingredient pre-workout supplement on resting energy expenditure and exercise performance in recreationally active females. J Int Soc Sports Nutr. 2018;15:1.

25. Walsh AL, Gonzalez AM, Ratamess NA, Kang J, Hoffman JR. Improved time to exhaustion following ingestion of the energy drink amino impact. J Int Soc Sports Nutr. 2010;7:14

26. Spriet LL. Exercise and sport performance with low doses of caffeine. Sports Med. 2014;44(Suppl 2):S175-84

27. Maughan RJ, Burke LM, Dvorak J, Larson-Meyer DE, Peeling P, Phillips SM, et al. IOC consensus statement: dietary supplements and the highperformance athlete. Br J Sports Med. 2018;52(7):439-55.

28. Southward K, Rutherfurd-Markwick KJ, Ali A. The effect of acute caffeine ingestion on endurance performance: a systematic review and meta-analysis. Sports Med. 2018;48(8):1913-28.

29. Pickering C, Grgic J. Caffeine and exercise: what next? Sports Med. 2019; 49(7):1007-30

30. Coull NA, Watkins SL, Aldous JW, Warren LK, Chrismas BC, Dascombe B, et al. Effect of tyrosine ingestion on cognitive and physical performance utilising an intermittent soccer performance test (iSPT) in a warm environment. Eur J Appl Physiol. 2015:115(2):373-86.

31. Woolf K, Manore MM. B-vitamins and exercise: does exercise alter requirements? Int J Sport Nutr Exerc Metab. 2006;16(5):453-84.

32. Sureda A, Pons A. Arginine and citrulline supplementation in sports and exercise: ergogenic nutrients? Med Sport Sci. 2012:59:18-28.

33. Goron A, Moinard C. Amino acids and sport: a true love story? Amino Acids. 2018;50(8):969-80.

34. Kreider RB, Kalman DS, Antonio J, Ziegenfuss TN, Wildman R, Collins R, et al International Society of Sports Nutrition position stand: safety and efficacy of creatine supplementation in exercise, sport, and medicine. J Int Soc Sports Nutr. 2017;14:18.

\section{Publisher's Note}

Springer Nature remains neutral with regard to jurisdictional claims in published maps and institutional affiliations. 